\title{
Preparation of micro oil droplets in micro-channel by digital jetting
}

\author{
Jinsong Zhang, Ge Xu, Chao Liu, Zhipeng Zhou and Liangwen Yan* \\ School of Mechatronic Engineering and Automation, Shanghai University, Shanghai City, China \\ Email: Iw_yan@staff.shu.edu.cn
}

Keywords: Two-phase slug flow; digital jetting; micro oil droplets; micro-channel

Abstract. In microfluidic chips, the micro droplet jetting is a key technology to control the droplet volume by micro-channel. The micro droplet in micro-channel is highly concerned on the two-phase or multi-phase slug flow, in which the two-phase streams can form two kinds of micro droplets steadily. This paper focused on the gas-oil two-phase slug flow to realize the digital jetting of the oil droplets. The flow rate of air was fixed to be $10 \mathrm{~mm}^{3} / \mathrm{s}$ and of oil was changed to be $4 \mathrm{~mm}^{3} / \mathrm{s}, 6 \mathrm{~mm}^{3} / \mathrm{s}$, $8 \mathrm{~mm}^{3} / \mathrm{s}$, and $10 \mathrm{~mm}^{3} / \mathrm{s}$. The jetting frequency of oil droplets increased in a linear relationship with the flow rate of oil while the flow rate of air was the same value. The volumes of oil droplets were similar to be $0.5 \mathrm{~mm}^{3}$ and the gas bubbles were surrounded by oil film.

\section{Introduction}

The ubiquitous fluids non-Newtonian fluids in nature, such as grease, paint, milk, have been widely used in chemical engineering, bio-chemical engineering, food processing, and medical engineering [1-3]. Many literatures reported all kinds of microfluidic devices to reduce the limitations on reagent requirements, enable faster reaction rates, reduce contamination, and be compatible with other functional components as micro total analysis systems [4]. Investigation of multiphase flows in microfluidic devices has been gaining lot of attention recently as a solution to improve and extend the performances of single phase flows. Multi-phase flow systems introduce a second immiscible flow to enhance mixing and transverse channel transport. The slug flow pattern takes some advantages on the large interfacial area, fast heat and mass transfer and reduced axial dispersion $[5,6]$.

The micro scale flow is more uniform and controllable than the traditional method of droplet generation [7]. The micro droplet jetting is to make droplets with a volume of nanoliter or below, and the formation of those microfluidic droplets typically occur at the instable phase-interfaces that subject to micro-scale interactions between fluids shear force and surface tension [8].

This paper investigated the two immiscible fluids, oil and gas, to form the slug flow pattern in micro-channel.

\section{Experimental}

The experimental system of gas-oil two-phase slug flow was set up as shown in figure 1 . The system had a gas control module, an oil control module, a nozzle and a computer. Both the flow rates of gas and oil had been controlled by the flow meters, which executed the commands delivering from the computer. During the experiments, the gas and oil streams were injected into the different micro-channel tubes, simultaneously and were mixed in the nozzle. Since the flow rates of oil and gas in the micro-channel were too fast to observe, the high speed camera was applied to observe the patterns of two-phase flow.

Figure 2 gives the structure of jetting nozzle, which contains two parts, the gas tube inside and the oil tube outside. The gas tube inserts into the oil tube and the stream oil enters the tube from the tube side, and the stream gas is injected the tube from the tube top. They take a mixture at the nozzle which is the rear part of oil tube before jetting. The end of gas tube and nozzle outlet is defined as the length of mixture chamber. 

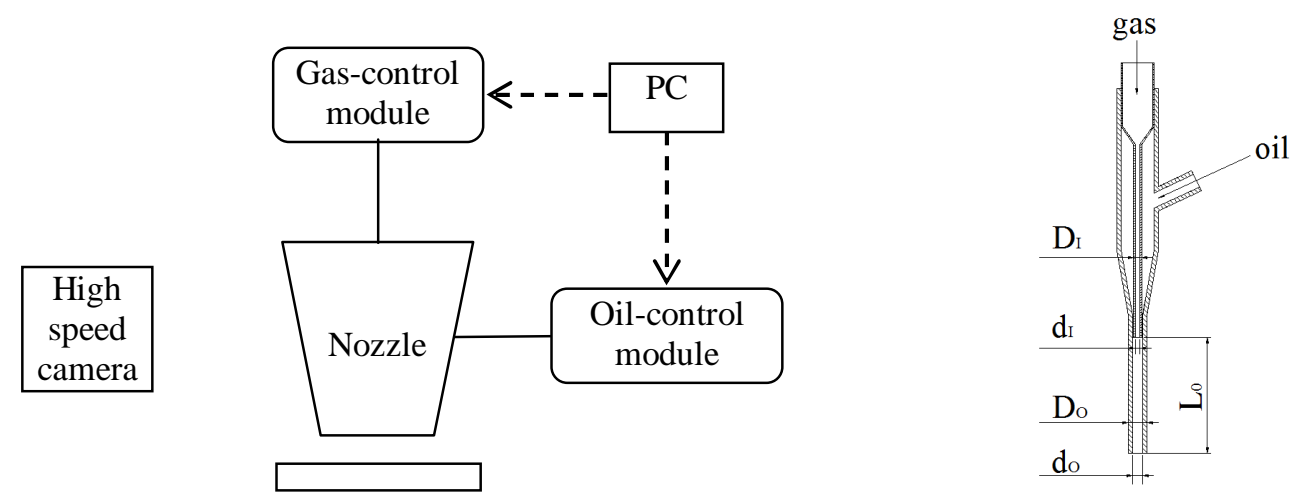

Fig. 1 The schematic diagram of experimental system Fig. 2 The schematic diagram of experiment

The parameters of experiment set up are listed in table1. In the table, $d_{o}$ is the internal diameter of oil tube, $D_{o}$ is the external diameter of oil tube, $d_{I}$ the internal diameter of gas tube, $D_{I}$ is the external diameter of oil tube, $L_{o}$ the length of mixture chamber.

\begin{tabular}{ccccc}
\multicolumn{5}{c}{ Tab. 1 The parameters of nozzle structure } \\
\hline$d_{o}$ & $D_{o}$ & $d_{I}$ & $D_{I}$ & $L_{o}$ \\
\hline 0.88 & 1.7 & 0.42 & 0.72 & 10.38
\end{tabular}

During the experiments, the experimental parameters and the physical properties of oil (Shell HX8 $5 \mathrm{~W}-40$ ) and gas are listed in table 2 . In the table $2, \rho$ is the density of material, $\mu$ is the dynamic viscosity, $\sigma$ is the surface tension.

Tab. 2 The experimental parameters and the properties of material

\begin{tabular}{ccccc}
\hline Materials & $\rho\left[\mathrm{g} / \mathrm{cm}^{3}\right]$ & $\mu[\mathrm{cp}]$ & $\sigma[$ dyn $/ \mathrm{cm}]$ & Flow Rates $\left[\mathrm{mm}^{3} / \mathrm{s}\right]$ \\
\hline Air & $1.205 \times 10^{-3}$ & 0.19 & -- & 10 \\
Oil & 0.851 & 64.9 & 63.4 & $4,6,8,10$ \\
\hline
\end{tabular}

\section{Results and discussion}

Figure 3 shows the photo of gas-oil slug flow captured by the high speed camera with the frame of $3000 \mathrm{fps}$. It is obviously that the transparent oil droplets have been separated by the black gas bubbles uniformly and steadily. The ruler (the division value $=1 \mathrm{~mm}$ ) in left can be used to measure the length of oil droplets and gas bubbles.

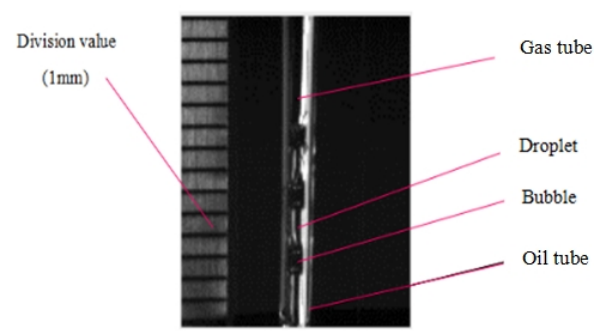

Fig. 3 The slug flow of gas and oil phases

Fixing the gas flow rate to be $10 \mathrm{~mm}^{3} / \mathrm{s}$ and changing the oil rate from $4 \mathrm{~mm}^{3} / \mathrm{s}$ to $10 \mathrm{~mm}^{3} / \mathrm{s}$, the gas-oil two-phase slug flow are still presented as following photos (Fig. 4).
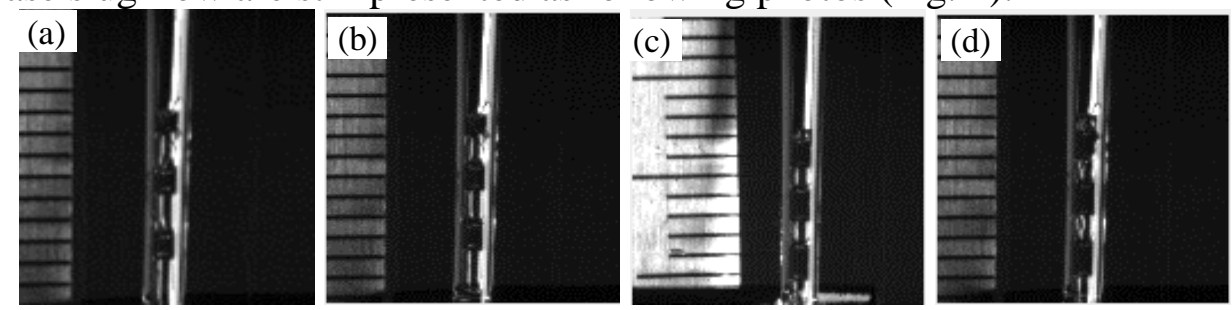

Fig. 4 The gas-oil two-phase slug flow with the different oil flow rates and the same gas flow rate, $q_{g}=10 \mathrm{~mm}^{3} / \mathrm{s}$, (a) $q_{o}=4 \mathrm{~mm}^{3} / \mathrm{s}$, (b) $q_{o}=6 \mathrm{~mm}^{3} / \mathrm{s}$, (c) $q_{o}=8 \mathrm{~mm}^{3} / \mathrm{s}$, (d) $q_{o}=10 \mathrm{~mm}^{3} / \mathrm{s}$.

The ratio of flow rate $(\varphi)$ between the oil and gas is 


$$
\varphi=\frac{q_{o}}{q_{g}}
$$

where $q_{o}$ is the flow rate of oil, $q_{g}$ is the flow rate of air.

The jetting frequency of droplets and bubbles $(f)$ can be expressed

$$
f=\frac{n}{t}
$$

Where, $n$ is the number of droplets, $t$ is time.

As shown in figure 5, $f$ has a linear relationship with the $\varphi$ increasing, while the gas flow rate is constant of $10 \mathrm{~mm} 3 / \mathrm{s}$. The maximum jetting frequency is about $19 \mathrm{~Hz}$ in it.

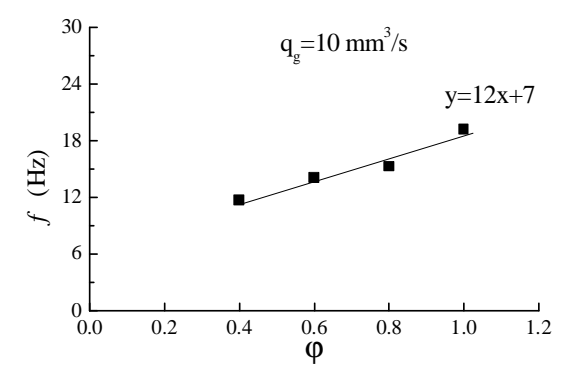

Fig.5 $\varphi$ versus $f\left(q_{g}=10 \mathrm{~mm}^{3} / \mathrm{s}\right)$

The droplet volume $V_{o}$ is

$$
V_{o}=\frac{q_{o}}{f}
$$

The droplet volume under different ratio of flow rate between oil and gas has been calculated in table 4. The tendency of droplet volume is proportional to the ratio of flow rate between the oil and gas. And the volume of oil droplets is similar to $0.5 \mathrm{~mm}^{3}$.

Tab.3 The droplet volume with the same flow rate of gas

\begin{tabular}{ccccc}
\hline$V_{o}\left[\mathrm{~mm}^{3}\right]$ & 0.3419 & 0.4255 & 0.5229 & 0.5208 \\
\hline$\varphi$ & 0.4 & 0.6 & 0.8 & 1.0 \\
\hline
\end{tabular}

In fact, there is an oil film at the interface between the bubble and wall in micro-channel. By the way, the bubbles are surrounded by the oil films to be Taylor shape. To simplify the thickness of oil film, the Taylor bubble is considered to be a cylinder shape.

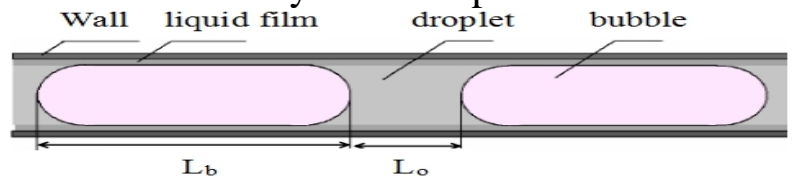

Fig. 6 The shape of slug flow in micro-channel

The bubble volume $\left({ }^{V_{b}}\right)$ is

$$
V_{b}=\frac{q_{b}}{f} \text { and } V_{b}=\pi r_{b}^{2} L_{b}
$$

The different $L_{b}$ are measured and listed in table 5 .

Tab. $4 L_{b}$ with different $\varphi$

\begin{tabular}{ccccc}
\hline$L_{b}(\mathrm{~mm})$ & 1.6 & 1.4 & 1.1 & 1.0 \\
\hline$\varphi$ & 0.4 & 0.6 & 0.8 & 1.0 \\
\hline
\end{tabular}

The bubble radius is calculated in figure 7 . It is true that the bubble radius is less than the inner wall radius. This can be attributed to the thin layer of oil film at the surface of inner wall. 


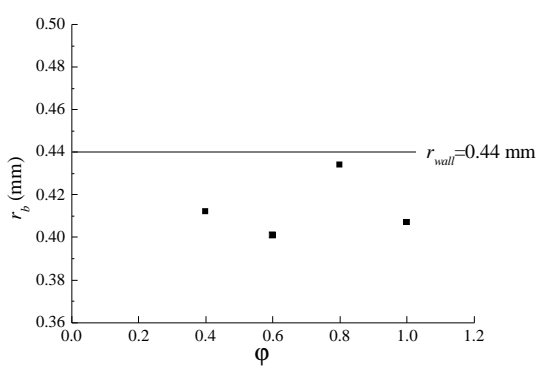

Fig.7 the diameter of bubbles

\section{Conclusions}

This paper studied the experiment of gas-oil two-phase slug flow in micro channel. The experiment structure had an inner gas tube and an outer oil tube. The gas and oil streams entered the tubes simultaneously and mixed at the nozzle to form the gas-oil two-phase slug flow steadily. Some conclusions can been drawn as following.

(1) The jetting frequency of droplets is in a positive linear relationship with the increase of the flow rate of oil. The maximum jetting frequency is about $19 \mathrm{~Hz}$ in experiments.

(2) The tendency of droplet volume is proportional to the increase of ratio of flow rates between oil and gas. And the volume of oil droplets is similar to $0.5 \mathrm{~mm}^{3}$.

(3) The bubble radius is less than the inner wall radius. This can be attributed to the thin layer of oil film at the surface of inner wall.

\section{Acknowledgements}

This article is sponsored by Natural Science Foundation of Shanghai (15ZR1416400).

\section{References}

[1]. Taotao Fu, Lijuan Wei, Chunying Zhu, Youguang Ma, Flow patterns of liquid-liquid two-phase flow in non-Newtonian fluids in rectangular microchannels, Chemical Engineering and Processing: Process Intensification, 2015(91): 114-120.

[2]. Chanyoot Keepaiboon, Somchai Wongwises, Two-phase flow patterns and heat transfer characteristics of R134a refrigerant during flow boiling in a single rectangular micro-channel, Experimental Thermal and Fluid Science, 2015 (66): 36-45.

[3]. Sung Chan Cho, Yun Wang, Two-phase flow dynamics in a micro channel with heterogeneous surfaces, International Journal of Heat and Mass Transfer 2014 (71): 349-360

[4]. Pinchuan Chen, Changwei Pan, Yulin Kuo, Performance characterization of passive micromixer with dual opposing strips on microchannel walls, Chemical Engineering and Processing: Process Intensification, 2015(93): 27-33.

[5]. Yuehao Li, Dawit G. Yamane, Shuning Li, Sanchita Biswas, Rupesh K. Reddy, Jost S. Goettert, Krishnaswamy Nandakumar, Challa S.S.R. Kumar, Geometric optimization of liquid-liquid slug flow in a flow-focusing millifluidic device for synthesis of nano materials, Chemical Engineering Journal, 2013(217): 447-459.

[6]. Pinchuan Chen, Yuchieh Chen, Chinmao Tsai, Microfluidic chip for rapid mixing and uniform distribution of multiple reagents using commercial pipettes, Microelectronic Engineering, 2016(150): 57-63.

[7]. Zhaomiao Liu, Likun liu, Feng shen, Experimental study on liquid-liquid two phase flow in different Y-angle of convection Y-junctions micro channels, 2014,46(2): 209-216.

[8]. Jiusheng Chen, Jiahuan Jiang, Droplet Microfluidic Technology: Mirodroplets Formation and Manipulation, Chinese Journal of analytical chemistry, 2012, 40(8): 1293-1300. 